Cite this: J. Mater. Chem. B, 2014, 2, 1706

Received 20th September 2013 Accepted 11th January 2014

DOI: $10.1039 / c 3 t b 21300 e$ www.rsc.org/MaterialsB

\section{Site specific immobilization of a potent antimicrobial peptide onto silicone catheters: evaluation against urinary tract infection pathogens $\uparrow$}

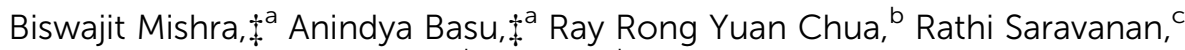 \\ Paul Anantharajah Tambyah, ${ }^{d}$ Bow Ho, ${ }^{b}$ Matthew Wook Chang ${ }^{\text {e }}$ \\ and Susanna Su Jan Leong*ef
}

Bacterial colonization of urinary catheters is a common problem leading to Catheter Associated Urinary Tract Infections (CAUTIs) in patients, which result in high treatment costs and associated complications. Due to the advantages of antimicrobial peptides (AMPs) compared to most other antimicrobial molecules, an increasing number of AMP-coated surfaces is being developed but their efficacy is hindered by suboptimal coating methods and loss of peptide activity upon surface tethering. This study aims to address this issue by employing a methodic approach that combines a simple selective chemical immobilization platform developed on a silicone catheter with the choice of a potent AMP, LasioglossinIII (Lasio-III), to allow site specific immobilization of Lasio-III at an effective surface concentration. The Lasio-III peptide was chemically modified at the $N$-terminal with a cysteine residue to facilitate cysteinedirected immobilization of the peptide onto a commercial silicone catheter surface via a combination of an allyl glycidyl ether (AGE) brush and polyethylene glycol (PEG) based chemical coupling. The amount of immobilized peptide was determined to be $6.59 \pm 0.89 \mu \mathrm{g} \mathrm{cm}^{-2}$ by Sulfo-SDTB assay. The AMPcoated catheter showed good antimicrobial activity against both Gram positive and negative bacteria. The antimicrobial properties of the AMP-coated catheter were sustained for at least 4 days postincubation in a physiologically relevant environment and artificial urine and prevented the biofilm growth of $E$. coli and E. faecalis. Adenosine tri-phosphate leakage and propidium iodide fluorescence studies further confirmed the membranolytic mode of action of the immobilized peptide. To the best of our knowledge, this is the first proof-of-concept study that reports the efficacy of AMP immobilization by sulfhydryl coupling on a real catheter surface.

\section{Introduction}

Bacterial colonization and biofilm formation in body implanted devices are of grave concern for modern society ${ }^{\mathbf{1}}$ mainly due to

${ }^{a}$ School of Chemical and Biomedical Engineering, Nanyang Technological University, 62 Nanyang Drive, 637459 Singapore

${ }^{b}$ Department of Microbiology, Yong Loo Lin School of Medicine, National University of Singapore, 5 Science Drive 2, 117545 Singapore

'School of Materials Science and Engineering, Nanyang Technological University, 62 Nanyang Drive, 637553 Singapore

${ }^{d}$ Department of Medicine, Yong Loo Lin School of Medicine, National University of Singapore, $1 E$ Kent Ridge Road, 119228 Singapore

${ }^{e}$ Department of Biochemistry, Yong Loo Lin School of Medicine, National University of Singapore, 8 Medical Drive, 117597 Singapore. E-mail: Susanna.Leong@ SingaporeTech.edu.sg; bchslsj@nus.edu.sg; Fax: +65 6592 1190; Tel: +65 65928544 ${ }^{f}$ Singapore Institute of Technology, 10 Dover Drive, 138683 Singapore

$\dagger$ Electronic supplementary information (ESI) available. See DOI: $10.1039 / \mathrm{c} 3 \mathrm{tb} 21300 \mathrm{e}$

\$ Equal contribution. the associated risks of infection culminating in high treatment costs, longer hospital stays and development of drug resistance. ${ }^{2,3}$

Microbial colonization on dental, heart valve and bone implants $^{4-6}$ as well as catheters ${ }^{7-12}$ is now a common occurrence. To circumvent such complications, scientists have adopted several strategies to design antimicrobial biomaterials. These strategies can be broadly categorized as: (a) surfaces that repel adsorption of proteins and bacteria ${ }^{\mathbf{1 3}, 14}$ and (b) surfaces that are conjugated with antimicrobial materials to incur cell death. ${ }^{15}$ However, these strategies have several drawbacks including insufficient surface antimicrobial concentration due to the low affinity of the biomaterials towards antimicrobial compounds such as antibiotics, unspecific chemical reaction methods for conjugation, a narrow activity spectrum and surface cytotoxicity. ${ }^{16-19}$ Additionally, the use of antibiotics can lead to the development of multidrug resistant (MDR) strains in common pathogens, thereby necessitating the development of better alternatives. 
Antimicrobial peptides (AMPs) have shown immense potential as antimicrobial agents mainly because of their broad activity spectrum and ability to reduce the pace of microbial tolerance development. ${ }^{20-23}$ In recent years, they have been explored as coating candidates for developing antimicrobial biomaterials. ${ }^{16,25,26}$ Several AMPs have been coated successfully on a variety of substrates which include contact lenses, glass, titanium oxide, silicone, resin beads, and silicon surfaces. ${ }^{16,27-31}$ Nevertheless, the coating of AMPs is still challenged by suboptimal coating strategies leading to inadequate surface concentrations, tedious chemical reactions, loss of antimicrobial activities with non-specific immobilization chemistry culminating in changed orientations of the peptide molecules and/or associated host cell toxicities. ${ }^{\mathbf{1 6 , 3 2}}$ Another recent strategy involves controlled release of the AMP molecules from reservoir surfaces. However, in contrast to covalent peptide immobilization, the controlled release of peptides is often challenged by the high peptide concentration required to incur significant killing in each release, and the need for this to be sustained for a reasonable period of time..$^{33,34}$

To address these problems, there is an ardent need for a combination of an efficient AMP candidate and an effective surface tethering strategy that would impart the desired antimicrobial characteristics on the targeted biomaterial. This study reports the development of the first AMP-coated silicone catheter prototype that shows good antimicrobial characteristics under physiological conditions.

The antimicrobial potential of immobilized Lasioglossin-III (Lasio-III) was previously demonstrated on silicon wafers. ${ }^{31}$ Like other salt tolerant AMPs, ${ }^{35,36}$ Lasio-III was also found to be active at physiologically relevant salt concentrations and possesses a high cell selectivity index (CI), which merits its use as a coating candidate. Subsequently, in a separate study, ${ }^{32}$ an allyl glycidyl ether (AGE) brush chemistry platform was also developed by our group, which allowed another AMP candidate, polybia-MPI, to be immobilized at a high surface concentration. Synergizing these outcomes to develop an effective AMP-coated catheter prototype, this work reports site directed immobilization of an N-terminus cysteine-modified Lasio-III using a PEG spacer coupled to an AGE brush onto a commercial silicone Foley catheter surface. The combination of AGE and PEG was aimed at improving both the anti-adsorption and antimicrobial properties of the surface. The effect of $\mathrm{N}$ - and C-terminal cysteine modification of the Lasio-III peptide on the site directed immobilization strategy via sulfhydryl chemistry was also studied.

The performance of the AMP-coated catheter was determined by studying the antimicrobial activity of the catheter against Escherichia coli (Gram-negative bacteria) and Enterococcus faecalis (Gram-positive bacteria), which are prevalent in urinary tract infections. ${ }^{37}$ Anti-biofilm and other in vitro properties of the peptide-immobilized catheter were also investigated.

\section{Materials and methods}

Chemically synthesized peptides were obtained from GL Biochem, Shanghai, China at a purity of $>90 \%$. All other chemicals were obtained from Sigma Aldrich, unless specified otherwise. Bacterial strains used in this study were Escherichia coli (ATCC8739), Pseudomonas aeruginosa (ATCC9027), Staphylococcus aureus (ATCC6538) and Enterococcus faecalis (ATCC29212).

\section{Antimicrobial activity and mechanistic investigations of Lasio-III in solution form}

The antimicrobial activity of soluble Lasio-III was studied by determining the minimal inhibitory concentration (MIC), minimal haemolysis concentration (MHC), and outer membrane permeabilization (NPN dye uptake) of the peptide. Structural studies were performed by circular dichroism (CD) spectroscopy and isothermal titration calorimetry (ITC) according to previously reported methods. ${ }^{31}$ ITC methods are detailed in the ESI (Materials and methods section) $+\dagger$

\section{Circular dichroism (CD) spectroscopy}

The secondary structure of Lasio-III variants in the presence and absence of SDS (sodium dodecyl sulphate) and DPC (dodecylphosphocholine) lipid micelles and trifluoroethanol (TFE) was determined using a Chirascan ${ }^{\mathrm{TM}}$ Circular Dichroism Spectrometer (Applied Photophysics Limited, U.K.). Peptide concentrations for CD studies were fixed at 100 $\mu \mathrm{g} \mathrm{mL}^{-1}$ in $10 \mathrm{mM}$ phosphate buffer ( $\mathrm{pH}$ 7.2). The final concentration of the lipid micelles was $10 \mathrm{mM}$. The spectra of peptide solutions contained in a $0.01 \mathrm{~cm}$ path length quartz cuvette (Hellma) were recorded at $25{ }^{\circ} \mathrm{C}$ from 190 to $240 \mathrm{~nm}$ using a bandwidth of $1 \mathrm{~nm}$ and averaged over three scans. Data were collected over a step size of 0.5 and a time constant of $0.5 \mathrm{~s}$. Baseline scans were obtained using the same parameters for samples containing buffer, micelles and vesicles only, and were subtracted from the respective data scans with peptides.

\section{Immobilization of CysLasio-III on silicone catheter surface}

Silicone Foley catheters (123616A, BARD) were purchased from National University Hospital Singapore, which comprised a 2 way silicone elastomer coat. The catheters were cut into $0.5 \times$ $1.0 \mathrm{~cm}^{2}$ rectangular pieces and treated with argon plasma for 5 min (March PX-500TM) at $300 \mathrm{~W}$ power. The surfaces were then incubated with allyl glycidyl ether (AGE) for 60 min under UV light to allow self-polymerization and brush formation on the silicone surfaces. The AGE-functionalized catheter surfaces were washed thoroughly with ethanol and reacted with ethylene-diamine for $18 \mathrm{~h}$ at room temperature (RT). Following this, the catheters were washed with DMF (dimethyl formamide) and further reacted with $0.5 \mathrm{mg} \mathrm{mL}^{-1}$ NHS$\mathrm{PEG}_{12}-$ maleimide in DMF for $4 \mathrm{~h}$ at RT. After washing with DMF and PBS ( $\mathrm{pH} 7.3$ ), the catheter samples were treated with $0.5 \mathrm{mg} \mathrm{mL}{ }^{-1}$ CysLasio-III in PBS ( $\mathrm{pH}$ 7.3) for $3 \mathrm{~h}$ at RT. Unbound peptides were removed by thorough rinsing with DI water. The detailed scheme of the reaction is illustrated in Fig. 1. 


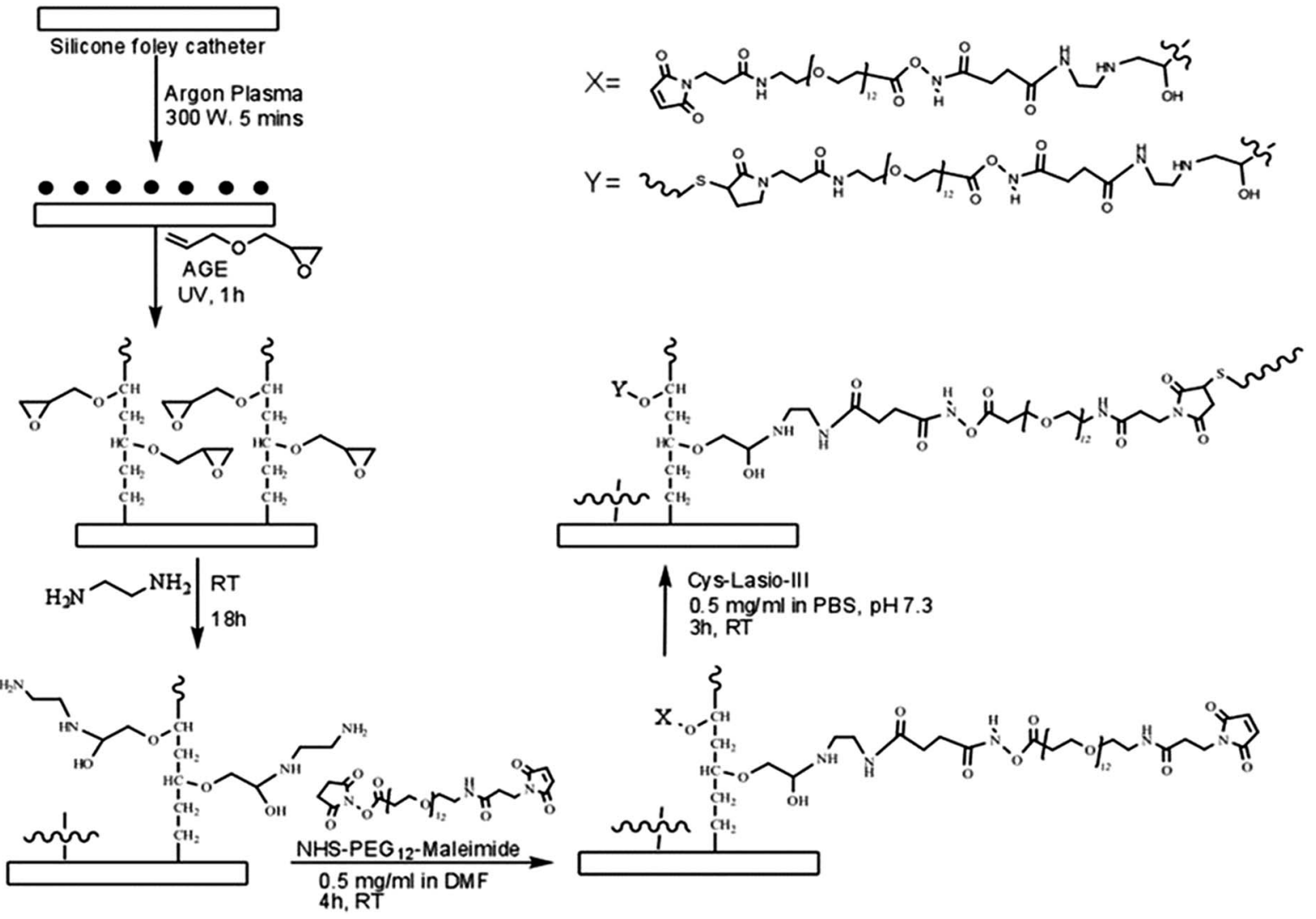

Fig. 1 Overall reaction scheme employed for immobilization of CysLasio-III onto the silicone catheter surface.

\section{Surface characterization of CysLasio-III-immobilized catheter}

The AGE- and peptide-functionalized silicone surfaces were air dried using a stream of nitrogen and characterized by $\mathrm{X}$ ray photoelectron spectroscopy (XPS) and attenuated total reflectance-infrared (ATR-IR) spectroscopy.

\section{X-ray photoelectron spectroscopy (XPS)}

Elemental analysis for the determination of successive reaction steps on the silicone surface was performed using an X-ray photoelectron spectrometer (Axis-ULTRA, Kratos) equipped with an $\mathrm{Al} \mathrm{K} \alpha$ source operating at a power of $10 \mathrm{~mA}$, $15 \mathrm{kV}$. Elements were identified from the survey spectra. High resolution spectra of carbon, nitrogen, oxygen and sulphur were recorded individually for elemental ratio comparisons.

\section{Attenuated total reflectance-infrared (ATR-IR) spectroscopy}

The successful grafting of CysLasio-III onto the catheter surfaces was verified by the presence of signature bonds by ATR-IR. The spectrum was recorded from $650 \mathrm{~cm}^{-1}$ to 4000 $\mathrm{cm}^{-1}$ by performing more than 32 scans with a Varian 3100 FTIR instrument equipped with a Diamond tip-ATR accessory unit.
Measurement of surface peptide concentration using sulfosuccinimidyl-4-o-(4,4-dimethoxytrityl) butyrate (SulfoSTDB)

A simple spectrophotometric assay was conducted to measure the surface peptide concentration. This method uses the high extinction coefficient value of a complex ion formed by the reaction of the surface amino groups and Sulfo-SDTB. The immobilized peptide concentration was determined using the supplier's instructions (Bioworld, USA). Briefly, $3.0 \mathrm{mg} \mathrm{mL}^{-1}$ of Sulfo-SDTB solution was prepared in DMF and then made up to $50.0 \mathrm{~mL}$ with $50 \mathrm{mM}$ sodium bicarbonate solution ( $\mathrm{pH}$ 8.5). 1.0 mL Sulfo-SDTB solution was added to the coated catheter samples and incubated for $1 \mathrm{~h}$ at RT. The samples were then washed twice with $5.0 \mathrm{~mL}$ of distilled water to remove any unreacted reactant, and immersed in $2.0 \mathrm{~mL}$ of perchloric acid for $30 \mathrm{~min}$. Finally, the absorbance at 498 nm was measured to detect the 4,4'-dimethoxytrityl cation (DMTr cation). The number of amine groups on the surface of each sample was quantified using the Beer-Lambert law with an extinction coefficient of $70000 \mathrm{M}^{-1} \mathrm{~cm}^{-1}$. The amount of peptides immobilized was calculated by calibrating with the predicted number of amines present in the peptide. ${ }^{28}$

\section{Anti-bacterial assay of CysLasio-III-immobilized catheters}

Anti-bacterial activity of the immobilized catheter samples was investigated according to the previously published ISO 22196 
protocol with minor modifications. ${ }^{31,38}$ In short, mid-exponential phase bacterial cells were adjusted to $10^{5} \mathrm{CFU} \mathrm{mL}^{-1}$ in LB medium. $20 \mu \mathrm{L}$ of the cell suspension was added to each well of a six-well culture plate which contained coated catheter samples to allow the inocula to surround the peptide-tethered surface. The plates were incubated at $37^{\circ} \mathrm{C}$ for $2 \mathrm{~h}$, followed by washing with $2 \mathrm{~mL}$ of fresh LB broth. The viable cells in solution were then serially diluted and $20 \mu \mathrm{L}$ aliquots were spread on LB agar plates for CFU determination after $16 \mathrm{~h}$ incubation. Catheters coated with NHS-PEG $12-$ Mal moieties only served as the control surfaces.

\section{Anti-biofilm activity of peptide immobilized catheters}

Quantitative evaluation by biofilms by XTT [2,3-bis(2-methyloxy-4-nitro-5-sulfophenyl)-2 $\mathrm{H}$-tertazolium-5-carboxanilide] assay. Biofilm growth was performed on a $0.5 \mathrm{~cm}^{2}$ catheter sample placed inside flat-bottomed polystyrene plates (Corning Inc. \#3799) using a reported protocol ${ }^{39}$ with slight modifications. Briefly, E. coli cells grown to the logarithmic phase were diluted to $10^{5} \mathrm{CFU} \mathrm{mL}{ }^{-1}$ in LB medium and $1.5 \mathrm{~mL}$ of this solution was further incubated with the catheter sample. Each sample was replicated for six wells $(n=6)$. Plates were incubated for $24 \mathrm{~h}$ at $37^{\circ} \mathrm{C}$ and analyzed for biofilm formation using the XTT assay according to the manufacturer's instructions (Sigma Aldrich, USA). Bacterial cells in the catheter without any peptide tethered served as the positive control, while uninoculated catheters were treated as the negative control.

\section{Live/dead biofilm staining}

After biofilm growth, the planktonic cells were removed by washing 3 times with neutral PBS buffer. They were stained with a LIVE/DEAD kit (Invitrogen Molecular Probes, USA) according to the manufacturer's instructions. The samples were examined with a confocal microscope (Olympus FV1000). For detection of SYTO 9 (green channel), the excitation wavelength was set at $488 \mathrm{~nm}$. For PI detection (red channel), an excitation wavelength of $561 \mathrm{~nm}$ was utilized. Images at both excitation wavelengths were captured and processed using FV10-ASW viewer software.

\section{Immersion-based killing assay on immobilized CysLasio-III on catheter surface}

Fresh E. coli cultures were prepared in LB medium to obtain $\mathrm{OD}_{600} \sim 0.5$. The final bacterial concentration of $1 \times 10^{6} \mathrm{CFU}$ $\mathrm{mL}^{-1}$ was obtained by suitable dilution. $1 \mathrm{~mL}$ of this diluted bacterial culture was incubated overnight with the CysLasio-IIItethered catheter samples $\left(0.5 \mathrm{~cm}^{2}\right)$ at $37^{\circ} \mathrm{C}$. Uncoated catheter samples were used as the positive control and uninoculated media as the negative control. Growth inhibitions were determined using $\mathrm{OD}_{600}$ measurements.

\section{Propidium iodide (PI) based membrane permeabilization assay of immobilized catheters}

Overnight E. coli cultures were sub-cultured in fresh $\mathrm{MH}$ medium and grown to the mid-log phase $\left(\mathrm{OD}_{600} \sim 0.5\right)$. Cells were then harvested by centrifugation $(10000 \times g, 5 \mathrm{~min})$ and washed thrice with fresh $10 \mathrm{mM}$ PBS (pH 7.3) to obtain a final cell concentration of $10^{8} \mathrm{CFU} \mathrm{mL}{ }^{-1} .600 \mu \mathrm{L}$ of this bacterial suspension containing $6 \mu \mathrm{M}$ PI was incubated with the CysLasio-III-immobilized catheter $\left(0.5 \mathrm{~cm}^{2}\right)$ samples for $1 \mathrm{~h}$ at $37^{\circ} \mathrm{C}$. The uncoated catheters were used as the negative control (considered $0 \%$ killed) and an equal volume of dead cells killed by $70 \%$ ethanol was used as the positive control (considered $100 \%$ killed). $100 \mu \mathrm{L}$ of the solution was then transferred into fresh 96 well plates for detection of PI fluorescence caused by intercalation of the dye into the DNA of dead cells. The corresponding signals were monitored using a multi-mode microplate reader (Synergy HT, BioTek Instruments, U.S.A.) at excitation and emission wavelengths of $485 \pm 20 \mathrm{~nm}$ and $600 \pm$ $20 \mathrm{~nm}$, respectively. The mean values for three triplicates were reported.

\section{Immobilized CysLasio-III stability testing by adenosine triphosphate (ATP) leakage assay}

To investigate the stability of the immobilized peptides, the catheter surfaces were incubated in PBS and Surine (synthetic urine) (obtained from Dyna Tek, USA) for four days. At least six catheter samples of $0.5 \mathrm{~cm}^{2}$ silicone surface were incubated with $3 \mathrm{~mL}$ PBS or Surine in a sterile 12 well culture plate and kept at RT inside a biosafety cabinet. Stability of the coated surfaces was evaluated by measuring the peptide's capability to cause ATP leakage in bacterial cells.

CysLasio-III-immobilized catheters in PBS and Surine were treated with $50 \mu \mathrm{L}$ of $10^{5} \mathrm{CFU} \mathrm{mL}{ }^{-1}$ of logarithmic phase $E$. Coli cells in PBS buffer. The cells were incubated for $30 \mathrm{~min}$ at $37{ }^{\circ} \mathrm{C}$ following which ATP leakage was quantified using the BacTiter-Glo kit (Promega) by measuring sample luminescence using a luminometer (Modulus Fluorometer 9200-000, Turner BioSystems, U.S.A.). PEG-coated catheters were used as the negative control while ATP leakage from bacteria treated with $1 \times$ MIC of the soluble peptide served as the positive control.

\section{Biocompatibility assay}

Evaluation using human red blood cells following haemolysis. The cytotoxicity of the immobilized catheter samples towards human red blood cells (hRBCs) was tested by incubating CysLasio-III-immobilized catheters with hRBCs (refer to the ESI†).

MTT (3-(4,5-dimethylthiazol-2-yl)-2,5-diphenyltetrazolium bromide $)$ assay. THP- 1 cells $\left(1 \times 10^{6}\right.$ cells $)$ were incubated in the presence of catheters (PEG-coated or PEG-CysLasio-III coated) and without catheters (control) in a final volume of $500 \mu \mathrm{L}$ culture medium, at $37{ }^{\circ} \mathrm{C}$ for $1 \mathrm{~h}$, with mixing at 15 min intervals. $20 \mu \mathrm{L}$ of cell suspension were then aspirated and examined by the trypan blue exclusion test under a light microscope. ${ }^{40-42}$ Catheters were removed and the MTT reagent was added to a final concentration of $0.5 \mathrm{mg} \mathrm{mL}^{-1}$ to cell suspensions, followed by $1 \mathrm{~h}$ incubation at $37^{\circ} \mathrm{C}$ for $1 \mathrm{~h}$, with mixing at 15 min intervals. The cells were lysed by adding $500 \mu \mathrm{L}$ of DMSO and incubated for $30 \mathrm{~min}$ at room 
temperature with regular mixing. Subsequently, acetic acid was added to a final concentration of $30 \% \mathrm{v} / \mathrm{v}$, and incubated for 15 min with regular mixing to ensure complete solubilization of the formazan salt. Light absorbance of the solubilized formazan salt at $570 \mathrm{~nm}$ was measured using an ELISA reader (Synergy HT, BioTek Instruments, U.S.A.). Parallel treatment of cells with $0.1 \%$ Triton-X-100 was carried out as total lysis control.

\section{Results and discussion}

\section{Feasibility study of cysteine-modified Lasio-III for tethering applications}

The choice of AMPs for immobilization applications should be governed by their killing mechanisms, where membraneinteracting peptides are hypothesized to be more effective compared to their 'internal target'-interacting counterparts. $^{23,24}$ For the latter class of peptides, the tethered peptide has to traverse across the whole cell membrane to reach cytoplasmic and nuclear targets, which could easily lead to non-specific binding and increase the peptide's minimum inhibitory concentration. Lasio-III was chosen as a peptide candidate for this study because of its potency and membrane targeting properties. ${ }^{31}$ A site specific sulfhydryl coupling reaction was employed to achieve unidirectional peptide immobilization. ${ }^{28-31}$ Cysteines were added to either the $\mathrm{N}$ or $\mathrm{C}$ terminal of the peptide, and the cysteine-modified peptides are known as CysLasio-III and Lasio-IIICys, respectively. The antimicrobial activity of CysLasio-III and Lasio-IIICys toward CAUTI relevant pathogens including E. coli, S. aureus, P. aeruginosa and E. faecalis was investigated (Table 1). The high antimicrobial potency of CysLasio-III, indicated by its submicromolar MIC, was comparable to that of the wild type peptide while Lasio-IIICys demonstrated a decrease in antimicrobial activity.

Incorporation of the cysteine residue increases the hydrophobicity of Lasio-III from 53\% to 56\% (calculated from the antimicrobial peptide database), ${ }^{21}$ leading to its increased haemolytic activity (Table 1 ). The increased haemolytic tendency observed for CysLasio-III is in agreement with that reported in previous studies, where the increased hydrophobicity in peptides is well correlated with enhanced haemolytic activity. ${ }^{\mathbf{4 4}}$ A series of peptide variants which were engineered based on DFTamp1 and Temporin-PTa templates

Table 1 Antimicrobial activity and cell selectivity index of Lasio-III cysteine variants against different bacteria

\begin{tabular}{lllll}
\hline & \multicolumn{2}{l}{ MIC of the peptide $(\mu \mathrm{M})$} & \\
\cline { 2 - 4 } Bacterial strains & Lasio-III $^{31}$ & CysLasio-III & Lasio-IIICys & CI $^{a}$ \\
\hline E. coli & 1.75 & 1.75 & 1.75 & 40.34 \\
S. aureus & 1.75 & 1.75 & 7.0 & 40.34 \\
P. aeruginosa & 3.5 & 7.0 & 14 & 10.08 \\
E. faecalis & ND & 0.9 & ND & 78.44
\end{tabular}

${ }^{a}$ The cell selectivity index of CysLasio-III(CI) was calculated using the formula $\mathrm{CI}=2 \times \mathrm{MHC}_{50} / \mathrm{MIC},{ }^{43}$ where the MHC value of CysLasio-III was found to be $35.3 \mu \mathrm{M}$; ND: not determined. showed increased cell selectivity index with decreasing retention time on a reverse phase HPLC analysis, which correlates with decreasing peptide hydrophobicity. ${ }^{\mathbf{4 3 , 4 4}}$ Despite an increased haemolytic tendency, the low MIC value of CysLasio-III contributes to the peptide's high CI value, which is a measure of the peptide's high selectivity toward bacterial cells. $^{2,31,43}$

As it is desirable for the immobilized peptide to possess membranolytic property, the peptide's ability to permeabilize the bacterial outer membranes by the NPN dye uptake assay was also investigated. The NPN dye molecules are hydrophobic and do not fluoresce in a hydrophilic environment. However they generate strong fluorescence signals upon entering a hydrophobic milieu like the outer bacterial membrane. ${ }^{45}$ Intact bacterial cells do not absorb the dye but if the peptide interacts with their outer membrane, it creates a passage for the NPN molecules to enter and fluoresce. Fig. 2A shows clear evidence of the interaction of NPN with the outer membrane. The enhanced fluorescence observed with the system containing the peptide and bacterial cells (relative to that obtained from polymyxin-B treated cells) clearly indicates outer membrane-peptide interactions. Amongst all the peptides, CysLasio-III showed the highest fluorescence reading indicating its higher efficiency in membrane binding.

The CD spectra of the peptides in the presence of negatively charged SDS (Fig. 2B) and zwitterionic DPC (Fig. 2C) micelles were also recorded. A clear dip in the CD spectrum was observed at 208 and $222 \mathrm{~nm}$ for all the three peptides, and it is most evident in CysLasio-III, indicating helicity development when in contact with the membrane mimicking lipid models (Fig. 2B and C) and tri-fluoro ethanol (TFE) (ESI Table S1 and Fig. $\mathrm{S} 1 \dagger$ ).

Additionally, the binding affinity of the peptides with DPC micelles was also quantitated under isothermal conditions. Fig. 2D shows the thermodynamic profile of the incubation of DPC with CysLasio-III in the upper panel and the corresponding fitted curve below. The peptide-DPC micelle interaction was characterized as an exothermic reaction with a binding constant $K_{\mathrm{a}}$ of $16.0 \times 10^{7} \mathrm{M}^{-1}$. The enthalpy change, $\Delta H$, of the reaction was determined to be $-10.93 \mathrm{kcal} \mathrm{mol}^{-1}$. For all the three peptides, the results are in close agreement with the structure and binding behavior reported for other bee venom peptides like melittin, ${ }^{\mathbf{3 1 , 4 6}}$ but the binding potential of CysLasio-III was found to be the highest (Table 2). Our data suggest that in contrast to the $\mathrm{C}$-terminal modified peptide, $\mathrm{N}$-terminal addition of cysteine increases the membrane binding potential of Lasio-III. As Lasio-III is naturally amidated at the C-terminal, it is plausible that the substitution of C-terminal amidation with cysteine modification may have perturbed the structural stabilization of the peptide via hydrogen bonding of the amide group with some other internal main chain residues. ${ }^{44}$ This possible loss of structural stability may contribute towards lowering the inherent membrane binding properties of native Lasio-III. CysLasio-III retained all the desired characteristics of the parent peptide and was used for site directed immobilization studies on silicone catheters. 


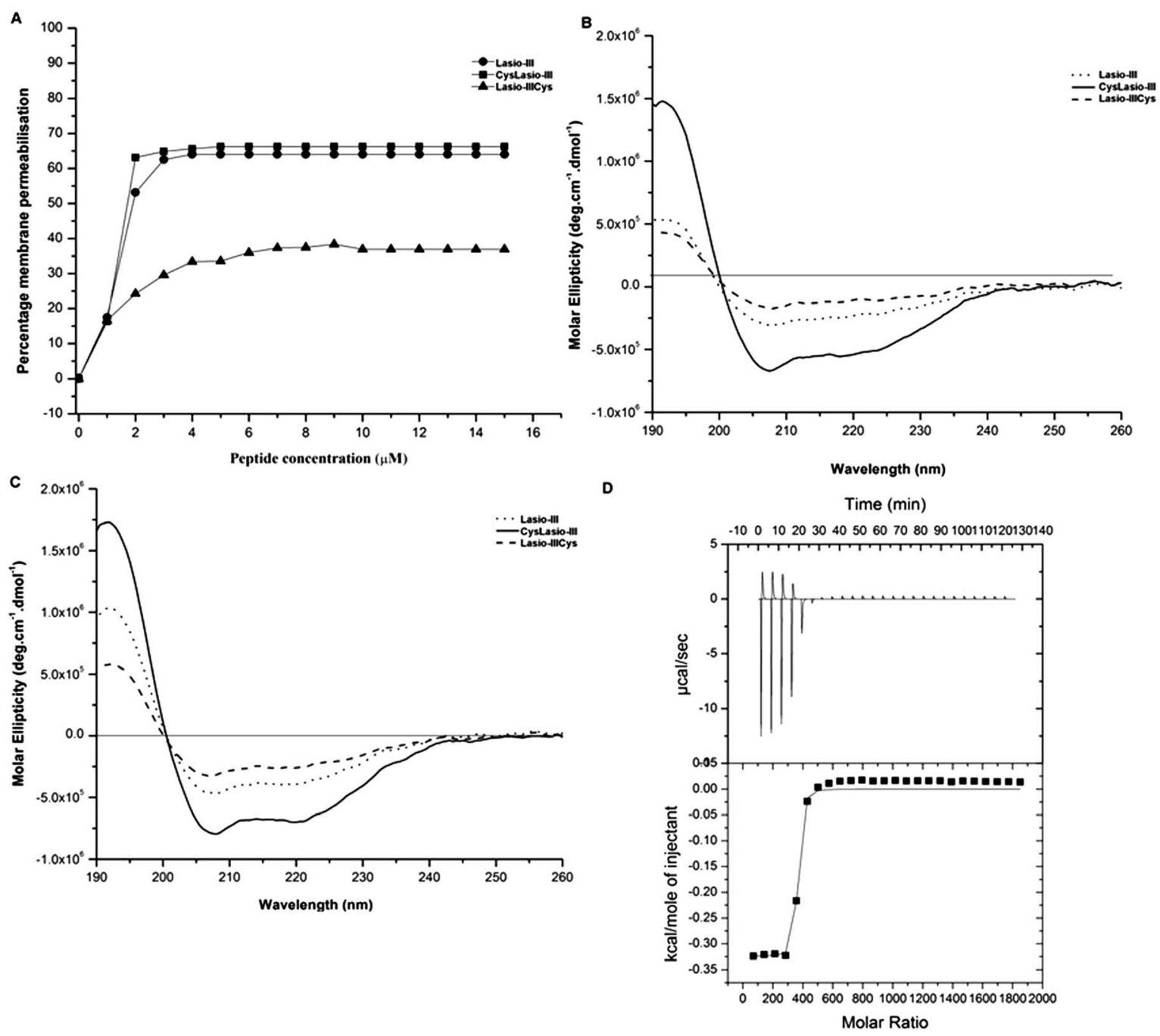

Fig. 2 In vitro characterization of the mechanisms of action of soluble Lasio- III ${ }^{31}$ and cysteine-modified variants. (A) Outer membrane permeabilization is shown by the uptake of the NPN dye at $410 \mathrm{~nm}$, with the results expressed relative to polymyxin-B. (B) CD spectra of the peptide samples interacting with negatively charged SDS and (C) zwitterionic DPC micelles. (D) ITC profile obtained from the interaction of CysLasio-III with DPC micelles. The interaction-profile with DPC micelles is presented in the upper panel while the curve fitting is shown in the bottom panel.

\section{Development of a peptide immobilization platform on silicone catheters}

A CysLasio-III immobilization platform was next developed on silicone catheter surfaces. The two pre-requisites set for peptide immobilization were the need for (i) the immobilized peptides to freely interact with the bacterial membranes and (ii) the catheter surface to have anti-adhesive properties to inhibit bacterial adsorption. An AGE brush layer was attached on the catheter surface, which was coupled to a bifunctional PEG

Table 2 ITC comparison of Lasio-III peptide variants

\begin{tabular}{lclll}
\hline Peptide & $K_{\mathrm{a}}\left(\mathrm{M}^{-1}\right)$ & $\begin{array}{l}\Delta H \\
\left(\mathrm{kcal} \mathrm{mol}^{-1}\right)\end{array}$ & $\begin{array}{l}\Delta G \\
\left(\mathrm{kcal} \mathrm{mol}^{-1}\right)\end{array}$ & $\begin{array}{l}T \Delta S \\
\left(\mathrm{kcal} \mathrm{mol}^{-1}\right)\end{array}$ \\
\hline Lasio-III & $13.8 \times 10^{7}$ & -10.8 & -9.52 & -1.28 \\
CysLasio-III & $16.0 \times 10^{7}$ & -10.93 & -9.43 & -1.5 \\
Lasio-IIICys & $2.1 \times 10^{7}$ & -12.56 & -10.7 & -1.86
\end{tabular}

linker. The polymerized AGE brush is advantageous due to its amphiphilic properties which contribute to an anti-adhesive surface. Fig. 1 shows a schematic for the immobilization chemistry. First, surface-radicals were generated on the Silicone Foley catheters through argon plasma treatment, which provides the points of attachment for the AGE molecules. AGE incubation under UV allows its self-polymerization through a radical transfer mechanism to form a brush with exposed epoxy groups. AGE was chosen for this particular step for two main reasons: first, the epoxy groups are known to remain stable under low energy radiation whilst allowing the polymerization reaction, and second, the number of surface epoxy groups can be modulated by the AGE concentration as well as the polymerization time..$^{32,47}$ These tethered epoxy groups can react with primary amines via a ring opening reaction following a $\mathrm{SN}_{2}$ mechanism.

When treated with ethylene diamine, the AGE brush would be functionalized with free amines along their lengths (Fig. 1). 
The free amine groups were then allowed to react with the $N$ hydroxysuccinimide moieties within the $\mathrm{PEG}_{12}$ chain, which also carries a maleimide moiety at the other end. The maleimide functional group was chosen because it is known to react specifically with free thiol groups of CysLasio-III to form a thioether linkage. ${ }^{\mathbf{4 8 , 4 9}}$ This tethering strategy therefore allows site specific coupling of CysLasio-III via the cysteine residue such that the peptide remains free to interact with the target pathogens. Furthermore, the AGE brush coupled with the PEG spacer would also provide ample flexibility to the tethered peptide to retain its activity even in the immobilized form..$^{50}$

\section{Surface characterization of CysLasio-III-immobilized silicone catheter}

Surface characterization studies were performed on the silicone catheters at different stages of reaction using XPS and ATR-IR spectroscopy. Elemental composition analysis of the surface was done by XPS. Wide range spectra for carbon 1s, nitrogen $1 \mathrm{~s}$ and oxygen 1s confirmed the presence of respective elements as anticipated from the chemical reactions (ESI Fig. S2†). The atomic concentration of nitrogen was measured to quantitatively and qualitatively validate the proceedings of the reaction. Fig. 3A shows nitrogen 1s peaks for each reaction step. Table 3 shows the variation in the atomic content and the ratios of $\mathrm{C} / \mathrm{O}$ and $\mathrm{C} / \mathrm{N}$ during each stage of reaction. In brief, the control catheter does not contain any nitrogen but following the ethylene-diamine reaction, the atomic content of nitrogen increased to $3.5 \%$. Subsequent reaction with $\mathrm{NHS}-\mathrm{PEG}_{12}-\mathrm{Mal}$ reduced the atomic content of nitrogen to $1.29 \%$, which was expected due to the addition of carbon and oxygen atoms, and following the peptide coupling reaction the nitrogen content increased up to $1.82 \%$ coupled with detected sulfur. The observed trend for nitrogen $1 \mathrm{~s}$ at $400 \mathrm{eV}$ verifies the successful proceedings of the reaction at each stage. The individual elemental ratios shown in Table 3 also support these results.

Furthermore, ATR-IR spectrometry was employed to assess the incorporation of specific functional groups onto the silicone surfaces after each reaction (Fig. 3B). The broad peak observed at $3100-3600 \mathrm{~cm}^{-1}$ within the spectrum of the CysLasio-IIIimmobilized catheter surface corresponds to the presence of hydroxyl and amine moieties on the silicone surface derived from the epoxy ring opening reactions and peptide tethering.

For the untreated silicone sample, the strong peak observed at around $1090 \mathrm{~cm}^{-1}$ is due to the $\mathrm{Si}-\mathrm{O}-\mathrm{Si}$ stretching vibrations which presumably masked the vibrations of the ethereal linkages $\left(\mathrm{CH}_{2}-\mathrm{O}-\mathrm{CH}_{2}\right)$ expected at around $1100 \mathrm{~cm}^{-1}$ for the peptide-coated catheter. The peak observed at $1650 \mathrm{~cm}^{-1}$ corresponds to the amide I stretching vibration from the tethered peptides. Additionally, the peak at $1560 \mathrm{~cm}^{-1}$ corresponds to the amide II band from the immobilized peptide. These results provide strong evidence of the successful immobilization of CysLasio-III onto the silicone surface.

\section{Determination of tethered surface peptide concentration}

Several strategies have been employed to quantitatively estimate the peptide concentration on different surfaces ${ }^{51}$ including
A a

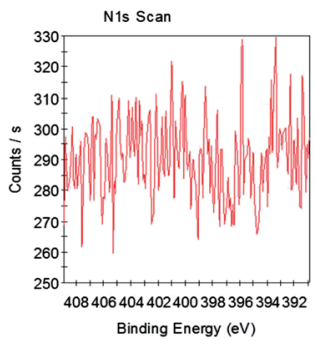

c

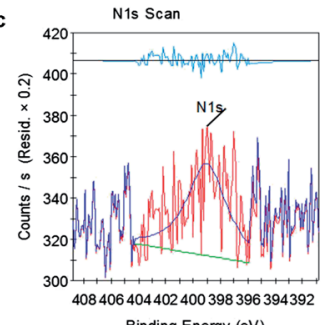

Binding Energy (eV)

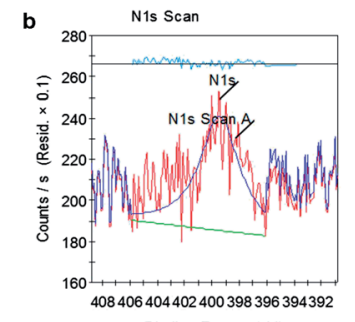

Binding Energy (eV)

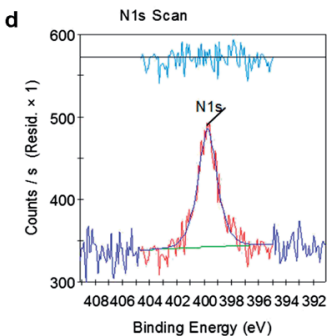

Binding Energy (eV)

B

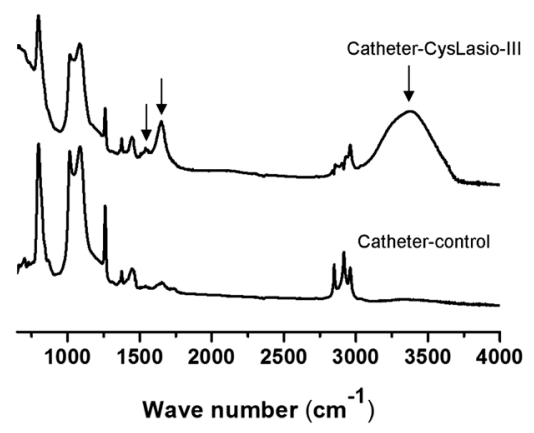

Fig. 3 Characterization of the CysLasio-III-immobilized catheter surface. (A) N1s spectra obtained from XPS analysis. (a) Cathetercontrol, (b) catheter-amine, (c) catheter-PEG $12-M a l$, and (d) catheter-CysLasio-III. (B) ATR-IR spectra of catheter-control and catheter-CysLasio-III.

ellipsometry, which was employed in our previous study to determine the peptide concentration on silicon surfaces. ${ }^{31,32}$ However, the use of such sophisticated physical techniques for determining surface peptide concentrations on soft polymeric materials including silicone remains challenged by their inability to reflect light. Additionally, the choice of analytical reagents also depends on the chemistry of the surface. In this study, an efficient and simple chemical method was employed

Table 3 Atomic percentage and ratio of elements obtained from XPS analysis of different reaction stages during peptide immobilization onto the silicone catheter surface

\begin{tabular}{lcclc}
\hline Element & $\begin{array}{l}\text { Catheter- } \\
\text { control }\end{array}$ & $\begin{array}{l}\text { Catheter- } \\
\text { amine }\end{array}$ & $\begin{array}{l}\text { Catheter- } \\
\text { PEG }_{12}-\text { Mal }\end{array}$ & $\begin{array}{l}\text { Catheter- } \\
\text { CysLasio-III }\end{array}$ \\
\hline C 1s & 47.56 & 43.34 & 48.9 & 49.65 \\
O 1s & 23.74 & 25.83 & 23.69 & 23.29 \\
N 1s & 0 & 3.5 & 1.29 & 1.82 \\
S 2p & 0 & 0 & 0 & 0.37 \\
Si 2p & 28.7 & 27.33 & 26.12 & 24.87 \\
C/N & 0 & 12.38 & 37.90 & 27.28 \\
C/O & 2.00 & 1.67 & 2.06 & 2.13
\end{tabular}


to quantify the surface immobilized peptides based on the number of peptide-derived free amine groups. ${ }^{52}$ The Sulfo-SDTB reagent specifically reacts with free amine groups to produce stoichiometric amounts of a chromophore, 4,4'-dimethoxytrityl cation (DMTr ion), which shows a strong absorbance at $498 \mathrm{~nm}$ and possesses an extinction coefficient of $70000 \mathrm{M}^{-1} \mathrm{~cm}^{-1}$. The amount of tethered peptides can then be derived using BeerLambert's law. Using this method, the amount of immobilized CysLasio-III on the silicone catheter surface was estimated to be $6.59 \pm 0.89 \mu \mathrm{g} \mathrm{cm}^{-2}$, which is comparable to those reported for other optimized platforms. ${ }^{25,28,53}$

\section{Bioactivity determination of the catheter-immobilized CysLasio-III}

In majority of cases, CAUTIs are initiated by the adherence of planktonic bacterial cells onto the biomaterial surface with subsequent progressive colonization and biofilm formation. ${ }^{54} \mathrm{It}$ is therefore important for our peptide-immobilized catheter to possess both bactericidal and anti-biofilm properties. The CysLasio-III-immobilized catheters possessed excellent antimicrobial properties toward E. coli and E. faecalis, resulting in $2 \log$ reduction kill of cells upon incubation (see ESI Table S2 $\dagger$ ). Anti-biofilm properties of the CysLasio-III-immobilized catheter were also assessed using XTT assay. Fig. 4 shows that the peptide-immobilized surface possessed significant anti-biofilm properties. A reduction of $60 \%$ and $40 \%$ biofilm was observed for E. faecalis and E. coli, respectively.

Fig. 5 shows the confocal microscopy images of the peptideimmobilized and PEG-functionalized catheters post-staining with the LIVE/DEAD kit. A high contrast of the green fluorescent colour obtained with PEG-coated control samples relative to the CysLasio-III-coated catheter is a clear indication of the high number of live cells present on the PEG-coated catheter surface compared to the peptide-coated catheter. The high contrast PI staining in CysLasio-III-coated catheters is evident of dead cells present on the surface of the peptide-immobilized catheters. Ongoing studies are underway to explore surface cleaning

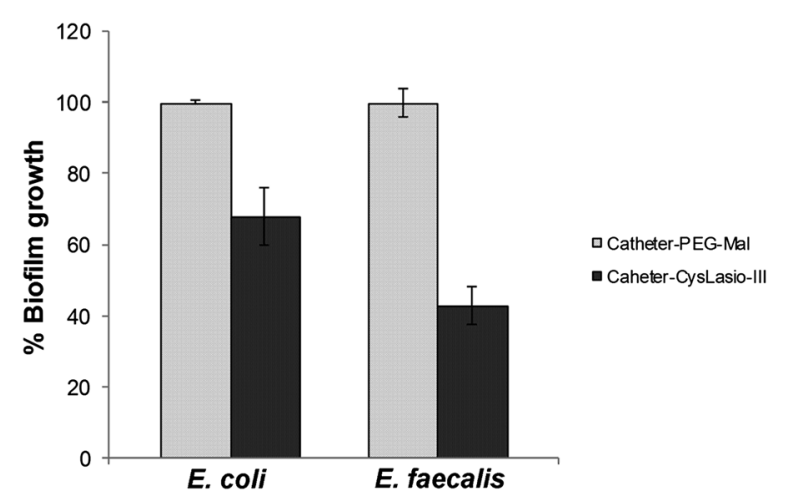

Fig. 4 Anti-biofilm activity of CysLasio-III-immobilized catheters $0.5 \mathrm{~cm}^{2}$ area of the immobilized catheter surface was used for antibiofilm activity determination. The controls (reaction on the catheters carried up to spacer attachment and devoid of any peptides) were considered to support $100 \%$ biofilm growth. ${ }^{31,32,39}$

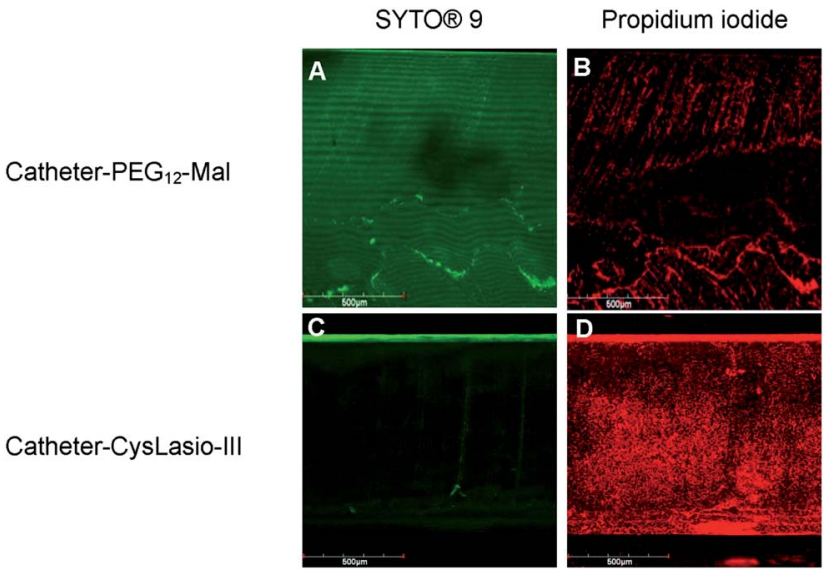

Fig. 5 Confocal laser scanning microscopy images of the $E$. faecalis biofilm stained by LIVE/DEAD assay. (A) Staining of the catheter$\mathrm{PEG}_{12}-$ Mal control with SYTO-9 and (B) propidium iodide. (C) Staining of catheter-CysLasio-III with SYTO-9 and (D) propidium iodide.

strategies to prevent multi-layer adsorption of dead cells onto the catheter surface.

The antimicrobial properties of the CysLasio-III-immobilized catheters were also investigated by an immersion based killing assay. $1 \mathrm{~mL}$ of $1 \times 10^{6} \mathrm{CFU} \mathrm{mL}^{-1}$ E. coli suspension was incubated with each peptide-immobilized catheter sample $\left(0.5 \mathrm{~cm}^{2}\right)$ overnight at $37^{\circ} \mathrm{C}$. Fig. $6 \mathrm{~A}$ shows the cell growth density after $18 \mathrm{~h}$ of incubation. The observed $\mathrm{OD}_{600}$ was comparable to that of the uninoculated media, which suggests
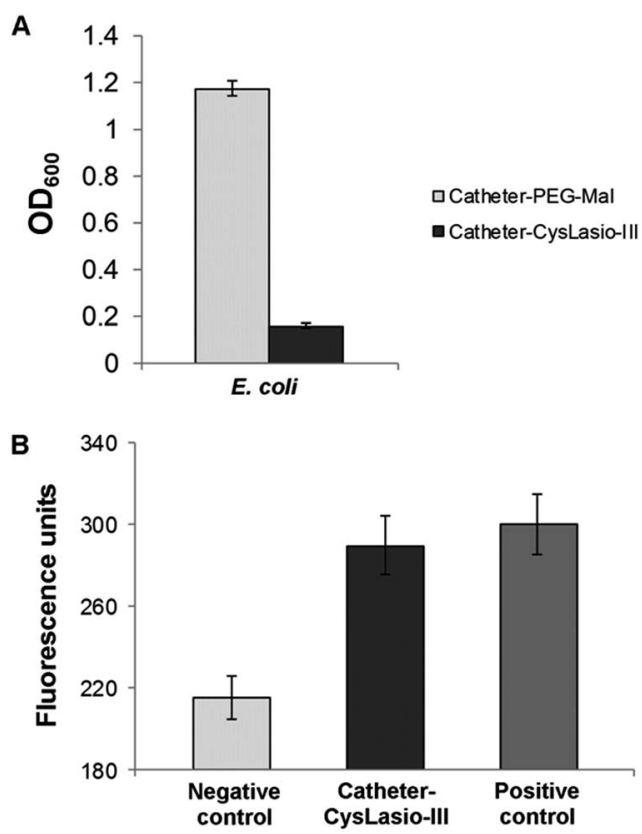

Fig. 6 (A) Immersion-based killing assay of the catheter-CysLasio-III, and (B) membrane permeabilization determination by PI intercalation against $E$. coli. The negative control comprised catheters functionalized with PEG spacers only while the positive control comprised nonviable cells incubated with $70 \%$ ethanol. 
that all the viable cells initially present in the peptide-coated catheters were killed. Additionally, a PI fluorescence assay was also employed to determine the extent of peptide-induced membrane permeabilization.

When the cell membrane is perturbed, the fluorescent dye enters the cells and intercalates with the DNA to exhibit fluorescence. Fig. 6B shows the PI fluorescence generated by the $E$. coli cells upon incubation with the CysLasio-III-immobilized catheters. The fluorescence intensity generated by the peptideimmobilized catheter sample is comparable to that of the positive control containing non-viable cells, which were killed using $70 \%$ ethanol, thereby providing clear evidence of membrane permeabilization.

\section{Stability and biocompatibility of CysLasio-III-immobilized catheters}

An important pre-requisite for AMP-coated catheters is the stability of the immobilized peptides. Since the urinary catheters will be impregnated in the urinary tract, the stability of catheter-CysLasio-III in artificial urine (Surine) was also tested. ${ }^{32}$ Fig. 7 shows the ATP leakage capability of the peptideimmobilized catheters toward E. coli cells in PBS and Surine environments after 4 days. Both PBS and Surine-incubated samples caused ATP leakage from the bacterial cells. However, the ATP leakage seems to be reduced with Surine, probably due to charge shielding effects incurred by the relatively high salt and ionic environment. Our results show that catheter-CysLasio-III can retain antimicrobial activity up to 4 days under physiological conditions but the stability can be compromised by salts and ions. Moreover, the ATP leakage assay further confirms the membranolytic mechanism of action of the immobilized catheters.

The biocompatibility of catheter-CysLasio-III was assessed by determining its haemolysis tendencies toward human red blood cells. Fig. 8A shows the interaction of the immobilized peptides with $5 \% \mathrm{v} / \mathrm{v}$ of hRBCs. The peptide-immobilized catheters showed $30 \%$ haemolysis which was nearly two fold less than that of the soluble peptide at a fixed peptide mass. This result agrees well with previous findings, where it has been

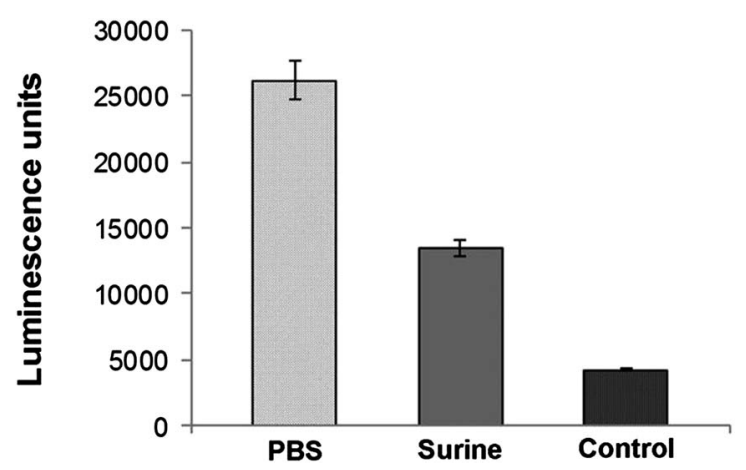

Fig. 7 Stability determination of catheter-CysLasio-III. Stability of the peptide-immobilized catheter surface by measuring ATP leakage after 4 days of incubation in PBS and Surine. The background signal obtained from the Bactiter reagent is kept as the control.

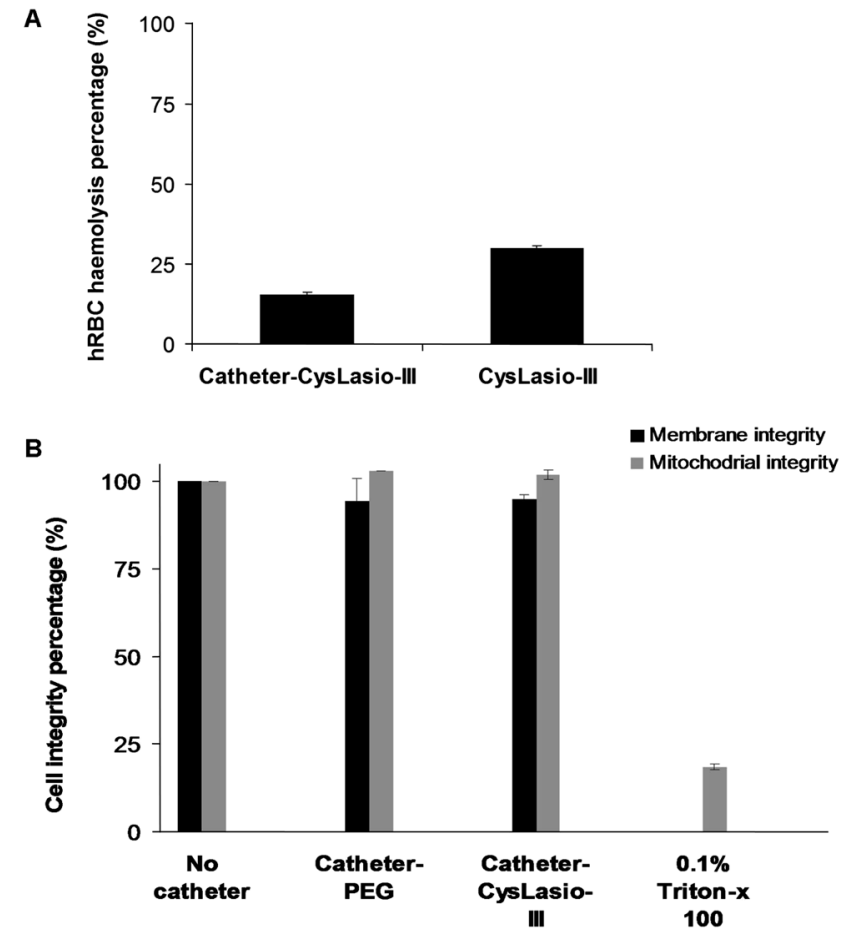

Fig. 8 Biocompatibility assays of catheter-CysLasio-III coupled with positive and negative controls. (A) The haemolysis potential of catheter-CysLasio-III with 5\% v/v hRBCs. The solution control contained the equivalent amount of soluble CysLasio-III peptide. (B) MTT assay against the THP-1 cell line.

shown that immobilization of the peptide leads to a reduced haemolytic effect. ${ }^{32}$ The MTT assay (Fig. 8B) also suggests reduced toxicity of both PEG-coated and CysLasio-III coated catheters towards other mammalian cell lines.

\section{Conclusion}

The efficacy of a cysteine-modified Lasioglossin peptide as a potential antimicrobial coating agent on silicone catheter surfaces was studied. Soluble CysLasio-III exhibits potent antimicrobial activity towards CAUTI-relevant pathogens such as $E$. coli and E. faecalis. CysLasio-III was successfully immobilized onto a commercial silicone catheter using a few simple chemical reaction steps. An AGE brush platform was developed to immobilize CysLasio-III using a PEG spacer and site directed coupling at the peptide N-terminal. Directional peptide immobilization enabled by sulfhydryl coupling is beneficial to maintain a specific orientation of the AMP which is likely to influence activity. ${ }^{31}$ Catheter-CysLasio-III demonstrated strong anti-adhesive properties, as exhibited by the anti-biofilm assay. The antimicrobial activity of the coated catheter showed potent antimicrobial activity against both $E$. coli and $E$. faecalis under physiologically relevant conditions, which shows its potential to inhibit bacterial proliferation in the urinary tract. Furthermore, the catheter stability tests revealed that the immobilized peptides were active for at least 4 days when submerged in Surine. The reduced toxicity of the immobilized catheter 
towards RBC and THP-1 cells demonstrates the peptide's good biocompatibility properties. The PI intercalation and ATP leakage assays confirmed the membranolytic mode of action of immobilized CysLasio-III. It has been postulated that immobilized peptides can induce electrostatic imbalance and changes in Donnan's equilibrium across the cytoplasmic membrane that may trigger self-death. ${ }^{55}$ This is the first proof-of-concept demonstration of site directed sulfhydryl immobilization of an AMP onto a urinary catheter surface that is effective against CAUTI-relevant pathogens while simultaneously showing good biocompatibility properties.

\section{Acknowledgements}

The authors acknowledge the assistance of Dr Li Peng, School of Chemical and Biomedical Engineering, NTU, Singapore, in operating the March PX-500TM plasma machine.

\section{References}

1 R. O. Darouiche, N. Engl. J. Med., 2004, 350, 1422-1429.

2 B. Mishra, V. Srivastava, R. Chaudhry, R. Somvanshi, A. Singh, K. Gill, R. Somvanshi, I. Patro and S. Dey, Amino Acids, 2010, 39, 1493-1505.

3 L. Zhao, P. K. Chu, Y. Zhang and Z. Wu, J. Biomed. Mater. Res., Part B, 2009, 91, 470-480.

4 P. R. Schmidlin, P. Muller, T. Attin, M. Wieland, D. Hofer and B. Guggenheim, J Appl. Oral Sci., 2013, 21, 48-55.

5 J. W. Costerton, L. Montanaro and C. R. Arciola, Int. J. Artif. Organs, 2005, 28, 1062-1068.

6 F. H. Furkert, J. H. Sorensen, J. Arnoldi, B. Robioneck and H. Steckel, Curr. Microbiol., 2011, 62, 1743-1751.

7 M. Harrod, C. P. Kowalski, S. Saint, J. Forman and S. L. Krein, BMC Health Serv. Res., 2013, 13, 151.

8 S. S. Lewis, L. P. Knelson, R. W. Moehring, L. F. Chen, D. J. Sexton and D. J. Anderson, Infect. Control Hosp. Epidemiol., 2013, 34, 744-747.

9 J. Rabideaux, Hospitals, 2013, 87, 14.

10 S. Saint, M. T. Greene, C. P. Kowalski, S. R. Watson, T. P. Hofer and S. L. Krein, JAMA Intern. Med., 2013, 173, 874-879.

11 P. A. Tambyah and J. Oon, Curr. Opin. Infect. Dis., 2012, 25, 365-370.

12 S. Saint, C. P. Kowalski, S. R. Kaufman, T. P. Hofer, C. A. Kauffman, R. N. Olmsted, J. Forman, J. BanaszakHoll, L. Damschroder and S. L. Krein, Clin. Infect. Dis., 2008, 46, 243-250.

13 G. M. Harbers, K. Emoto, C. Greef, S. W. Metzger, H. N. Woodward, J. J. Mascali, D. W. Grainger and M. J. Lochhead, Chem. Mater., 2007, 19, 4405-4414.

14 M. E. Buck, A. S. Breitbach, S. K. Belgrade, H. E. Blackwell and D. M. Lynn, Biomacromolecules, 2009, 10, 1564-1574.

15 F. Costa, I. F. Carvalho, R. C. Montelaro, P. Gomes and M. C. L. Martins, Acta Biomater., 2011, 7, 1431-1440.

16 M. Bagheri, M. Beyermann and M. Dathe, Antimicrob. Agents Chemother., 2009, 53, 1132-1141.
17 M. A. Gelman, B. Weisblum, D. M. Lynn and S. H. Gellman, Org. Lett., 2004, 6, 557-560.

18 M. F. Ilker, K. Nüsslein, G. N. Tew and E. B. Coughlin, J. Am. Chem. Soc., 2004, 126, 15870-15875.

19 K. Kuroda and W. F. DeGrado, J. Am. Chem. Soc., 2005, 127, 4128-4129.

20 K. A. Brogden, Nat. Rev. Microbiol., 2005, 3, 238-250.

21 G. Wang, X. Li and Z. Wang, Nucleic Acids Res., 2009, 37, D933-D937.

22 H. G. Boman, Annu. Rev. Immunol., 1995, 13, 61-92.

23 R. E. Hancock and R. Lehrer, Trends Biotechnol., 1998, 16, 82-88.

24 M. Bagheri, M. Beyermann and M. Dathe, Bioconjugate Chem., 2012, 23, 66-74.

25 G. Gao, D. Lange, K. Hilpert, J. Kindrachuk, Y. Zou, J. T. Cheng, M. Kazemzadeh-Narbat, K. Yu, R. Wang, S. K. Straus, D. E. Brooks, B. H. Chew, R. E. Hancock and J. N. Kizhakkedathu, Biomaterials, 2011, 32, 3899-3909.

26 M. Kazemzadeh-Narbat, J. Kindrachuk, K. Duan, H. Jenssen, R. E. Hancock and R. Wang, Biomaterials, 2010, 31, 95199526.

27 J. Peyre, V. Humblot, C. Methivier, J. M. Berjeaud and C. M. Pradier, J. Phys. Chem. B, 2012, 116, 13839-13847.

28 K. Lim, R. R. Chua, R. Saravanan, A. Basu, B. Mishra, P. A. Tambyah, B. Ho and S. S. Leong, ACS Appl. Mater. Interfaces, 2013, 5, 6412-6422.

29 M. Mohorcic, I. Jerman, M. Zorko, L. Butinar, B. Orel, R. Jerala and J. Friedrich, J. Mater. Sci.: Mater. Med., 2010, 21, 2775-2782.

30 M. D. Willcox, E. B. Hume, Y. Aliwarga, N. Kumar and N. Cole, J. Appl. Microbiol., 2008, 105, 1817-1825.

31 B. Mishra, A. Basu, R. Saravanan, L. Xiang, L. K. Yang and S. S. J. Leong, RSC Adv., 2013, 3, 9534-9543.

32 A. Basu, B. Mishra and S. S. Jan Leong, J. Mater. Chem. B, 2013, 1, 4746-4755.

33 I. Izquierdo-Barba, M. Vallet-Regí, N. Kupferschmidt, O. Terasaki, A. Schmidtchen and M. Malmsten, Biomaterials, 2009, 30, 5729-5036.

34 D. G. Desai, K. S. Liao, M. E. Cevallos and B. W. Trautner, J. Urol., 2010, 184, 2565-2571.

35 M. Pasupuleti, A. Schmidtchen, A. Chalupka, L. Ringstad and M. Malmsten, PLoS One, 2009, 4, e5285.

36 A. Majerle, J. Kidrič and R. Jerala, J. Antimicrob. Chemother., 2003, 51, 1159-1165.

37 L. E. Nicolle, Urologic Clinics of North America, 2008, vol. 35, pp. 1-12.

38 D. Kowalczuk, G. Ginalska and J. Golus, Int. J. Pharm., 2010, 402, 175-183.

39 S. N. Dean, B. M. Bishop and M. L. van Hoek, BMC Microbiol., 2011, 114.

40 T. Mosmann, J. Immunol. Methods, 1983, 65, 55-63.

41 W. Strober, Curr. Protoc. Immunol., 2001, appendix 3appendix 3B.

42 S. Tsuchiya, M. Yamabe, Y. Yamaguchi, Y. Kobayashi, T. Konno and K. Tada, Int. J. Cancer, 1980, 26, 171-176.

43 J. Menousek, B. Mishra, M. L. Hanke, C. E. Heim, T. Kielian and G. Wang, Int. J. Antimicrob. Agents, 2012, 39, 402-406. 
44 B. Mishra and G. Wang, J. Am.Chem. Soc., 2012, 134, 1242612429.

45 B. Loh, C. Grant and R. E. Hancock, Antimicrob. Agents Chemother., 1984, 26, 546-551.

46 R. Saravanan, A. Bhunia and S. Bhattacharjya, Biochim. Biophys. Acta, Biomembr., 2010, 1798, 128-139.

47 B. Thierry, M. Jasieniak, L. C. P. M. De Smet, K. Vasilev and H. J. Griesser, Langmuir, 2008, 24, 10187-10195.

48 G. T. Hermanson, Bioconjugate Techniques, Academic Press, San Diego, 1996.

49 A. Basu and S. S. J. Leong, Anal. Biochem., 2011, 418, 155-157.

50 M. Gabriel, K. Nazmi, E. C. Veerman, A. V. N. Amerongen and A. Zentner, Bioconjugate Chem., 2006, 17, 548-550.
51 F. Zaera, Chem. Rev., 2012, 112, 2920-2986.

52 R. K. Gaur and K. C. Gupta, Anal. Biochem., 1989, 180, 253258.

53 G. Gao, J. T. Cheng, J. Kindrachuk, R. E. Hancock, S. K. Straus and J. N. Kizhakkedathu, Chem. Biol., 2012, 19, 199-209.

54 C. R. Arciola, D. Campoccia, P. Speziale, L. Montanaro and J. W. Costerton, Biomaterials, 2012, 33, 5967-5982.

55 K. Hilpert, M. Elliott, H. Jenssen, J. Kindrachuk, C. D. Fjell, J. Korner, D. F. Winkler, L. L. Weaver, P. Henklein, A. S. Ulrich, S. H. Chiang, S. W. Farmer, N. Pante, R. Volkmer and R. E. Hancock, Chem. Biol., 2009, 16, 58-69. 